\title{
Review: paracetamol reduces pain in osteoarthritis but is less effective than NSAIDs
}

Zhang W, Jones A, Doherty M. Does paracetamol (acetaminophen) reduce the pain of osteoarthritis? A meta-analysis of randomised controlled trials Ann Rheum Dis 2004;63:901-7.

Is paracetamol efficacious for treatment of osteoarthritis (OA)?

\section{METHODS}

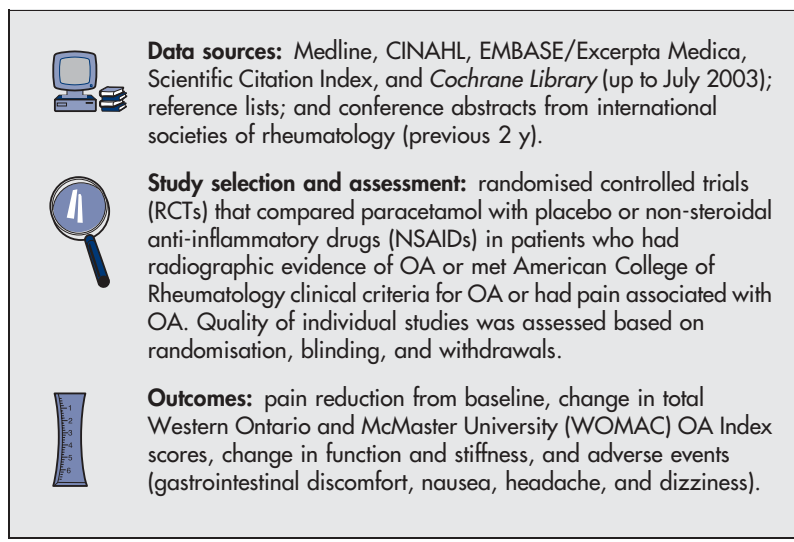

\section{MAIN RESULTS}

10 trials $(\mathrm{n}=2144)$ met the selection criteria. Comparison groups were placebo (4 comparisons) and NSAIDs ( 11 comparisons). Mean age ranged from 56-64 years. Median study length was 6 weeks (range 1 wk to 2 y). 7 trials used a fixed dose of paracetamol, 4000 $\mathrm{mg} / \mathrm{day}$, and 3 used dosages of 2000, 2600, and $3000 \mathrm{mg} /$ day, respectively. NSAIDs included diclofenac, celcoxib, ibuprofen, rofecoxib, and naproxen, and dosages varied by drug. Paracetamol $v$ placebo. Meta-analysis of 2 trials showed that patients who received paracetamol had greater pain reduction than those who received placebo (table), but groups did not differ for overall WOMAC scores (table), functioning (table), stiffness (table), or side effects. NSAIDs $v$ paracetamol. Meta-analysis of 8 trials ( 11 comparisons) showed that patients who received NSAIDs had greater pain reduction and better WOMAC scores than those who received paracetamol (table). NSAIDs were associated with greater gastrointestinal discomfort, but groups did not differ for nausea, headache, or dizziness.

\section{CONCLUSIONS}

Paracetamol reduces osteoarthritis pain more than placebo but does not affect functioning or stiffness. Non-steroidal anti-inflammatory drugs are more effective than paracetamol for pain reduction but are associated with greater gastrointestinal discomfort

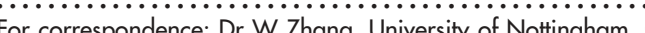
Nottingham, UK. weiya.zhang@nottingham.ac.uk

Source of funding: Arthritis Research Campaign.

\section{Commentary}

Treole he systematic reviews by Zhang et al and Lin et al examined the effectiveness of oral and topical treatments for osteoarthritis. These treatments have been recommended by the American College of Rheumatology ${ }^{1}$ and in treatment guidelines developed in Europe and the UK. ${ }^{23}$ The use of paracetamol and topical NSAIDs must be even more closely examined in light of the withdrawal of rofecoxib from the market. In a thorough review, Lin et al concluded that topical NSAIDs were superior to placebo in reducing pain and improving function, although effects waned after 2 weeks. The authors also noted that effects differed based on the specific drug. Comparisons of topical and oral NSAIDs were based on only 1 or 2 studies, and comparisons of various topical agents were not done in this review. The well done systematic review by Zhang et al examined the efficacy of paracetamol for reducing osteoarthritis pain and concluded that paracetamol is effective and safe for osteoarthritis pain relief. A similar review published in the Cochrane Library found that oral NSAIDs are more effective, but perhaps less safe, than paracetamol in reducing osteoarthritis pain. ${ }^{4}$

The results of these 2 reviews are important to nurses who work with geriatric patients, community health nurses, and advanced practice nurses who might prescribe or recommend treatments for patients with osteoarthritis. Historically, paracetamol and topical treatments have been the mainstay of osteoarthritis therapies. Approximately 5 years ago, a new category of drugs (cyclooxygenase-2 [COX 2] inhibitors) came onto the market and virtually revolutionised osteoarthritis care. Clinical trials showed that COX 2 inhibitors safely reduced osteoarthritis pain with short term use. ${ }^{5}$ Rofecoxib, the first COX 2 inhibitor to be approved, was recently voluntarily withdrawn by the manufacturer because of increased cardiovascular complications with long term use. Although several other COX 2 inhibitors are still available, long term use of these agents for osteoarthritis will no doubt be scrutinised.

Healthcare providers can use the results of these reviews to recommend topical NSAIDs for short term treatment of temporary osteoarthritic flares only, as they do not appear to be useful as chronic therapies. Paracetamol is an effective treatment and should be recommended as a first line treatment. Healthcare researchers should focus future efforts on comparing various topical drugs and comparing topical and oral NSAIDs to determine their relative efficacy, safety, and longevity of action. Cathy R Kessenich, RN, DSN, ARNP

1. Arthritis Rheum 2000;43:1905-15. University of Tampa, Tampa, Florida, USA

2 Grilli R, Magrini N, Penna A, et al.

4 Pendleton A, Arden N, Dougados M, et al. Ann Rheum Dis 2000;59:936-44. 2003;(2):CD004257.

5 Fine PG. J Pain 2002;3:272-83.

Paracetamol $v$ placebo or non-steroidal anti-inflammatory drugs (NSAIDs) for osteoarthritis (OA)*

\begin{tabular}{|c|c|c|c|c|}
\hline \multirow[b]{2}{*}{ Outcomes } & \multicolumn{2}{|c|}{ Paracetamol $v$ placebo } & \multicolumn{2}{|c|}{ NSAIDs $v$ paracetamol } \\
\hline & $\begin{array}{l}\text { Number of } \\
\text { comparisons (n) }\end{array}$ & Pooled effect size $(95 \% \mathrm{Cl})$ & $\begin{array}{l}\text { Number of } \\
\text { comparisons (n) }\end{array}$ & Pooled effect size $(\mathrm{Cl})$ \\
\hline Pain & $2(394)$ & $0.21(0.02$ to 0.41$) \dagger$ & $11(1641)$ & $0.20(0.10$ to 0.30$) \neq$ \\
\hline WOMAC OA score & $2(394)$ & $0.14(-0.06$ to 0.34$)$ & 4 (NA) & $0.30(0.17$ to 0.44$) \ddagger$ \\
\hline Function & Not assessed & Not assessed & 6 (NA) & $0.22(0.08$ to 0.34$) \ddagger$ \\
\hline Stiffness & Not assessed & Not assessed & 5 (NA) & $0.35(0.19$ to 0.50$) \ddagger$ \\
\hline
\end{tabular}

${ }^{*} \mathrm{NA}=$ not available. WOMAC = Western Ontario and McMaster University. $\mathrm{Cl}$ defined in glossary

†Significant result favouring paracetamol over placebo.

‡Significant result favouring NSAIDs over paracetamol. 